\title{
CHARACTERISATION OF MORPHOLOGY IN MUSCULUS SEMITENDINOSUS TENDON USED FOR ANTERIOR CRUCIATE LIGAMENT RECONSTRUCTION: A PILOT STUDY
}

\author{
Janis Davis Osipovs*, Mara Pilmane*, Modris Ciems** \\ * Riga Stradins University, Institute of Anatomy and Anthropology, Latvia \\ ** Traumatology and Orthopaedics Hospital, Riga, Latvia
}

\begin{abstract}
Summary
Introduction. Anterior cruciate ligament (ACL) rupture is very common in athletes. In the general population, incidence is approximately 33 per 100000 people.

Aim of the study. The aim of the study was the evaluation of morphological changes in the musculus semitendinosus tendon used for the reconstruction of $\mathrm{ACL}$ to understand the quality of most common for surgery used material.

Material and methods. The materials were obtained from four ACL autologous hamstring reconstruction surgeries. The tissue was stained with hematoxyllin and eosin and with immunohistochemical (IMH) staining of PGP9.5, VEGF, collagen I and collagen III. The material was evaluated with semiquantitative method.

Results. Routine staining showed practically unchanged tendon structure, with one exception when sclerotic blood vessels were observed in endotenon. Collagen III IMH demonstrated moderate to numerous positive collagen fibres in two cases, but in other two there were only few positive structures seen. Collagen I IMH showed few to moderate number of positive collagen fibres in all cases. In two cases, moderate number of PGP9.5 positive nerve fibres was observed and in two other cases occasional to few number of positive structures was detected. PGP 9.5 marked higher number of nerve fibres in peritenon than in endotenon. Numerous VEGF positive endotheliocytes were observed in two cases, but in two other cases VEGF positive endotheliocytes were occasional.

Conclusions. Tendon of musculus semitendinosus displays two patterns of distribution of tissue ischemia, neuropeptide containing innervation and collagen I and III. Collagen III is thought to be evaluated as a response of tendon to the ischemia and intensive innervation, while increase of collagen I probably is related to the relatively unchanged vascularity and innervation. The pattern of musculus semitendinosus tendon structural changes seems to be connected to the individual homeostasis in patients persisting before the usage of tendon for the reconstruction.
\end{abstract}

Keywords: Anterior cruciate ligament (ACL), orthopaedic surgery, musculus semitendinosus, tendon, tissue factor

\section{INTRODUCTION}

Anterior cruciate ligament (ACL) rupture is very common in knee. It is known that ACL injury is associated with some sports (e.g. soccer, basketball). Females have a higher risk of having ACL damage than men. It is estimated that females have approximately 3.7 times higher risk of ACL damage doing basketball and 2.7 times higher risk doing soccer (20). Increased incidence for females is thought to be associated with neuromuscular activation factors and hormonal factors, when highest ACL injury risk is during ovulatory phase (10). Incidence of ACL injury within athletes could be 1 on every 3000 to 4000 practise or game exposures, but in general population incidence is 29 to 38 per 100 000 people (16)

An ACL injury usually requires surgical treatment. For treatment, an autograft and allograft could be used. Very often surgeons use autografts from hamstring muscles, also bone-patellar tendon-bone grafts (5). However, frequent complications are persistent knee pain and extension loss. The reason for knee pain after ACL reconstruction is not known (15).

It is important to understand changes in normal histology of tendon in pathological condition. In normal tendon tenocytes are attached to extracellular matrix (ECM) which in tendon is mainly composed of collagen I ( $70 \%$ of dry mass). Collagen I fibres and tenocytes are encircled with connective tissue endotenon, peritenon, epitenon (with progenitor cell populations which can differentiate into vascular and nervous system cells). Collagen III is mainly associated with tendon reparation processes. Abundance of collagen III marks lower technical properties of tendon and could be a sign of degeneration. In very low quantities collagen V and VI could be also seen in tendon. Elastin also is component of ECM $(\sim 1 \%$ dry mass) and regulates adequate mechanical properties of tendon (23).

Surgical implant used in cruciate ligament reconstruction can vary depending on its structural 
and functional aberrations. It is known that reduced angiogenesis after tendon injury improves tendon regeneration. Tendons with reduced angiogenesis show better organized matrix, increased stiffness and higher Junge module - maximum stress resistance (24). In contrast, blood vessel formation and VEGF expression and high appearance of neural markers PGP9.5 and growth associated protein 43 (GAP43) are characterized by early painful tendinopathy. These changes also occur when tendon is damaged and afterwards tendon regeneration process is initiated ( 1 , 22, 29). Experiments with animals showed that older and physically inactive mice have decreased expression of ECM remodelling genes in the tendon and signs of calcification. Older and well-trained mice expressed more actively connective tissue growth factor, VEGF, decorin gene and thus signs of calcification are decreased (13). In the case of tendinopathy also tenocyte morphology can change, reduced fibroblast count and fibroblast "rounding" can occur (12).

Not very much is known about morphopathogenesis in regeneration of tendon. It is thought that important is to have high expression of well organised collagen with larger diameter fibril size. Altered collagen production from type I to III can accelerate tendon injury repair but also decreases mechanical properties of tendon and can predispose tendon rupture. After tendon injury collagen fibrils tend to be smaller in diameter, because of collagen III over expression $(9,14)$. Experiments with Achilles tendon demonstrated that three days after tendon injury interleukin 6 (IL-6), interleukin $1 \beta$ (IL-1 $\beta$ ), matrix metalloproteinase 9 (MMP9), matrix metalloproteinase 13 (MMP13), collagen III is increased, however on day seven collagen I is increased and after two weeks TGF- $\beta 1$ is increased and decreases collagen I/III ratio (7). TGF- $\beta 1$ induces differentiation of progenitor cells in tendon, increases collagen III expression and regulates collagen VI, which is important in fibrillogenesis, thereby accelerating tendon reparation processes. These accelerated reparation processes also are connected with higher VEGF expression $(8,21,28,30)$.

Some influence on the structure of tendon depends on the biomechanical strength so, knee joint has very interesting signalling pathway during motion - when there are movements in knee joint, then articulatory cartilage squeezes and interstitial fluid from cartilage gets into articulatory cavity. This interstitial fluid affects cells in knee joint, also cruciate ligament tenocytes, which afterwards stimulates expression of hyaluronan synthase l (HAS1), hyaluronan synthase 2 (HAS2), aggrecan, lubricin, matrix metalloproteinase 3 (MMP3), tissue inhibitor of metalloproteinase 3 (TIMP3) and TGF $\beta 1$, but inhibits expression of collagen I, versican, MMP2, TIMP2, tumor necrosis factor (TNF), and ILl $\beta$ (17). MMP and TIMP balance is important in maintaining structural integrity of ECM and aberrations could lead to disrupted ECM organisation regulation thereby causing ligament rupture $(2,6,11)$.

For reconstruction of ACL injury autografts are used more commonly, but allograft use is considered when patient refuse a usage of autografts or patient has multiple ligamentous injuries. Most commonly used implants are from patellar tendon, musculus semitendinosus or musculus gracilis tendons (18).

\section{AIM OF THE STUDY}

The aim of the study was to determine whether all obtained ACL transplants show similar immunohistochemical properties. If any differences between samples were noted, then evaluation of changes in the muscle semitendinosus tendon should be done to understand the quality of the most commonly used ACL surgical repair material.

\section{MATERIAL AND METHODS}

The materials used in this study were obtained from four surgical procedures of ACL autologous hamstring reconstruction, in which sample was collected as a small piece of the side of the graft during surgical procedure. The patients age varied from 26 to 55 . Samples obtained during surgical procedure were immediately fixated in transport test tube with stefanini and delivered to Riga Stradins University Anatomy and Anthropology institute (RSU AAI), where in Morphology laboratory tissue were prepared for routine histology staining (hematoxyllin (260830, Bio Optica) and eosin (4610106, Diapath)), and for immunohistochemical (IMH) staining of PGP9.5 (439273A, rabbit, 1:200, Zymed Laboratories, UK), VEGF (orb191500, rabbit, 1:100, Biorbyt, USA), collagen I (ab88147, mouse, 1:100, Abcam, UK), collagen III (242410, rabbit, 1:50, Quartett Immunodiagnostika, Germany). The material was evaluated with microscope and relative number of different markers was analysed with semiquantitative method (19). The labels were as followed: (0) - no positive structures are detectable in visual field; $(0 /+)-$ occasional positive structures seen in the visual field; $(+)$ - few positive structures seen in the visual field; $(++)$ - moderate number of positive structures seen in the visual field; $(+++)$ - numerous number of positive structures seen in the visual field; $(++++)-$ abundant number of positive structures seen in the visual field.

\section{RESULTS}

Routine staining showed practically unchanged tendon structure (Fig. 1), with one exception when sclerotic blood vessels were observed (Fig. 2). Some tendon regions demonstrated also oedema of tissue and different size of collagen fibre bundles in places here and there.

Collagen III demonstrated moderate to numerous positive structures in two cases, but in other two there were only few positive structures (Fig. 3). Collagen I showed similar number of positive structures in all cases and it was seen in few to moderate fibres (Fig. 4). Neural marker PGP 9.5 marked nerve fibres in endotenon and peritenon, with higher number of nerve fibres in peritenon. In two cases, a moderate number of PGP9.5 positive nerve fibres were observed and in two other cases occasional to few number of 
positive structures was seen (Fig. 5-6). Numerous VEGF positive endotheliocytes were observed in two cases but in two other cases VEGF positive endotheliocytes were occasional in number (Fig. 7).

\section{DISCUSSION}

Commonly, there were two patterns for factor expression in tendon. In two cases there were numerous number of VEGF positive endotheliocytes, but in other two cases VEGF positive endotheliocytes were seen only occasionally. The pattern with increased VEGF could be associated with extensive previous mechanical load which increases need for oxygenation and nutrient supplementation (27). It is known, that also hypoxia stabilises hypoxia-inducible factor (HIF), which is transcription factor for genes including VEGFA (4). Additionally, VEGF is important in endotheliocyte migration, cell survival and is known as a strongest endothelyocyte mitogen. VEGF positive endotheliocytes are associated with hypoxia and play role in angiogenesis $(4,27)$.

In two cases of our research there was moderate number of PGP9.5 positive nerve fibres, but in other two cases PGP9.5 positive structures were seen occasionally in peritenon. Earlier studies indicated that in tendon increased number of PGP9.5 positive nerve fibres is associated also with VEGF increase $(1,22,29)$. It is not exactly known what is the mechanism underlying this process. In our case, increased innervation could potentially be associated with tendon regeneration after previously underwent injury and/or response to heavy physical exercise and biomechanical changes (26). The second pattern with occasional PGP9.5 positive nerve fibres could be evaluation of normal structure of tendon.

Also collagen III showed two patterns of distribution, where moderate to numerous collagen III fibres were seen in two cases and in other two cases collagen III fibres were occasional. Collagen III is mainly associated with tendon reparation processes, where abundance of collagen III marks lower technical properties of tendon and could be a sign of degeneration (23). We may agree with this, because in our research increased collagen III pattern showed also signs of ischemia and increased innervations in surrounding connective tissue, but reversely occasional collagen III fibres of other pattern could be mark of normal tendon structure.

Researches evaluating tensile strength of regenerated and after operation residual tendon; showed that fibroblasts density highly increases right after injury, which is associated with reparation processes. Fibroblast density then decreased over the time and after 24 weeks, tensile strength of regenerated tissue is approximately $75 \%$ comparing to control. This leads to conclusion that tendon showing marks of regeneration is less resistant to mechanical load than tendon showing less marks of regeneration. Thereby collagen III produced by tendoblasts could potentially be beneficial marker to evaluate before predicting the potential strength of the implant (25).
In two cases there were few collagen I fibres and in the other two there was moderate number of collagen I fibres seen in endotenon and peritenon. Interestingly when decreased number of VEGF, PGP9.5 and collagen III positive structures was observed, then increased number of collagen I fibres was seen. In this case moderate number of collagen I fibres could be seen in unchanged tendon, but decreased amount of collagen I could be a mark of pathological changes. Previous studies report that collagen I is a main component of tendon structures (23). To maintain structural and functional integrity of a tendon, it is important to have balance between synthesis and breakdown of ECM. It is believed that regulation of maintaining balance is controlled by genetic factors. Unfortunately, this process is poorly understood. MMP plays an important role as colagenases in maintaining local homeostasis, any disregulation of MMP can affect structure of ECM (3).

At the end, tendon samples can be divided into two distinct patterns. The first pattern can be described with increased VEGF, PGP9.5, collagen III and decreased number of collagen I fibres. The second pattern shows occasional VEGF, PGP9.5, collagen III positive structures and more collagen I fibres than first pattern. The first pattern shows properties of hypoxia and tissue regeneration, while other patterns suggests none of these properties. This raises questions about the quality of implant material. Thus, we hypothesize that first pattern with regeneration marks could react differently after a significant period of time comparison to the second pattern, which is similar to the healthy tendon.

\section{CONCLUSIONS}

1. The tendon of musculus semitendinosus used for reconstruction of ACL rupture seems to display two patterns of distribution of tissue ischemia and neuropeptide containing innervation: indistinct and distinct.

2. Collagen I and III also show two patterns of distribution. Increased prevalence of collagen III is thought to be evaluated as a response of tendon to the ischemia and intensive innervation, while an increase of collagen I probably is related to the relatively unchanged vascularity and innervation.

3. The pattern of musculus semitendinosus tendon structural changes presumably could be connected to the individual homeostasis in patients persisting before the usage of tendon for the reconstruction as analysed in discussion section.

\section{ACKNOWLEDGEMENTS}

The authors would like to thank Dr. E.Ozols for the supplying of the material.

\section{Conflict of interest: None}




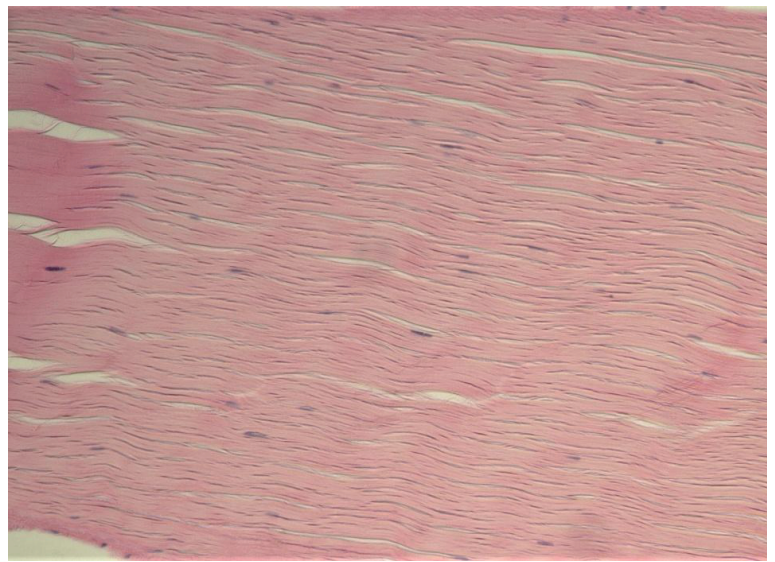

Fig. 1. Note regularly organised tendon collagen fibres of similar diameter in 26 years old patient material. Hematoxyllin and eosin, X100

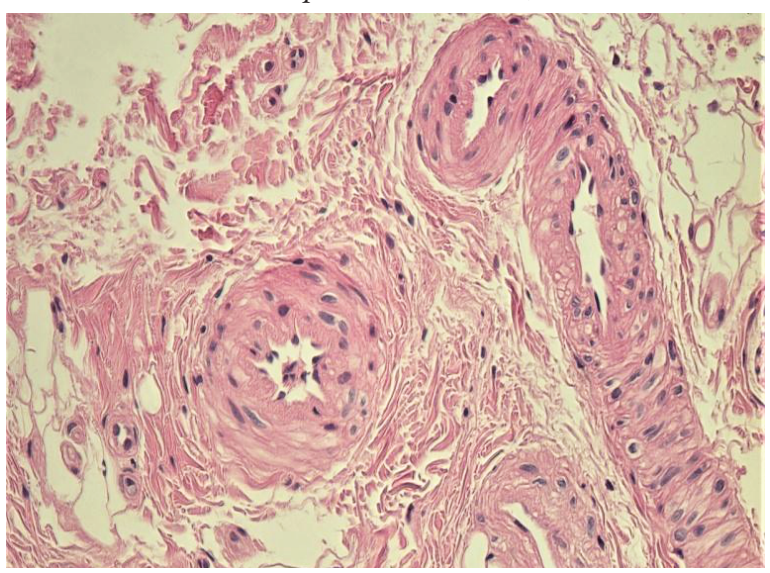

Fig. 2. Note sclerotic arteries in 31 years old patient in musculus semitendinosus peritenon. Hematoxyllin and eosin, X250

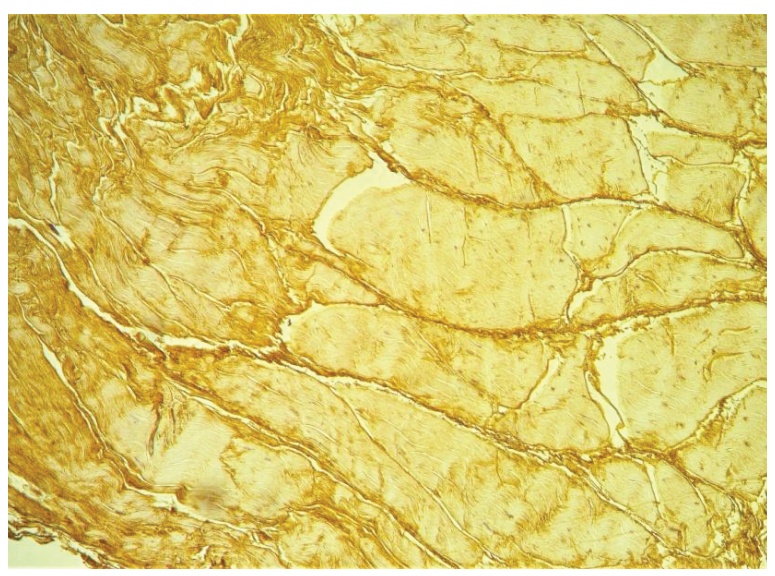

Fig. 3. Moderate to numerous number of collagen III positive structures seen in 31 years old patient in musculus semitendinosus material. Collagen III IMH, X100

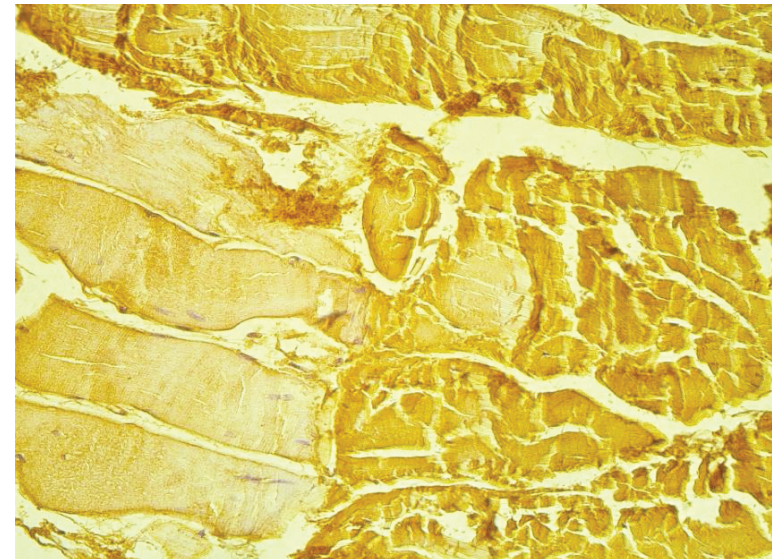

Fig. 4. Moderate number of collagen I positive structures in 38 years old patient in musculus semitendinosus material. Collagen I IMH, X200

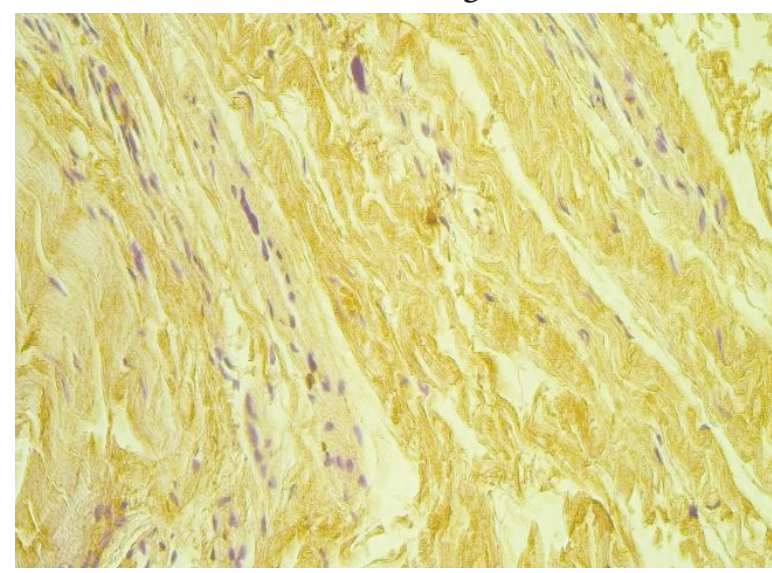

Fig. 5. Few PGP9.5 positive nerve fibres seen in musculus semitendinosus in 26 years old patient material. PGP9.5 IMH, X200

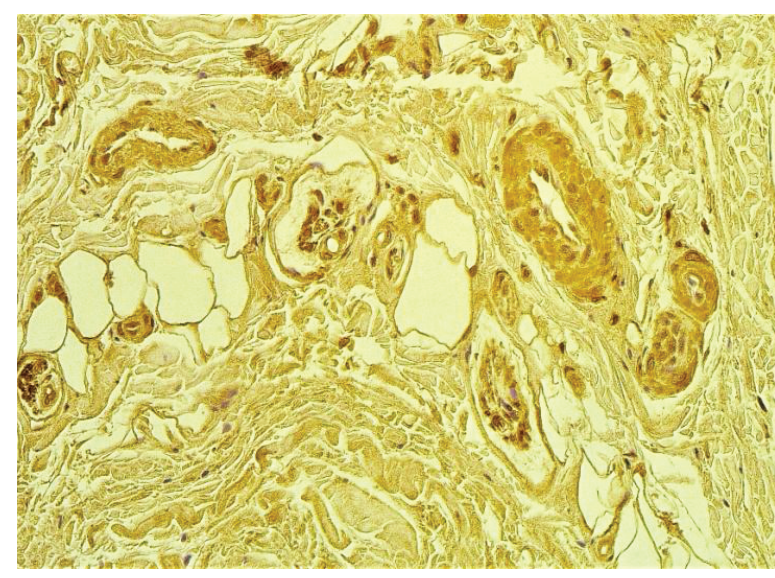

Fig. 6. Moderate number of PGP9.5 positive nerve fibres seen in musculus semitendinosus peritenon in 31 years old patients material. PGP9.5 IMH, X200 


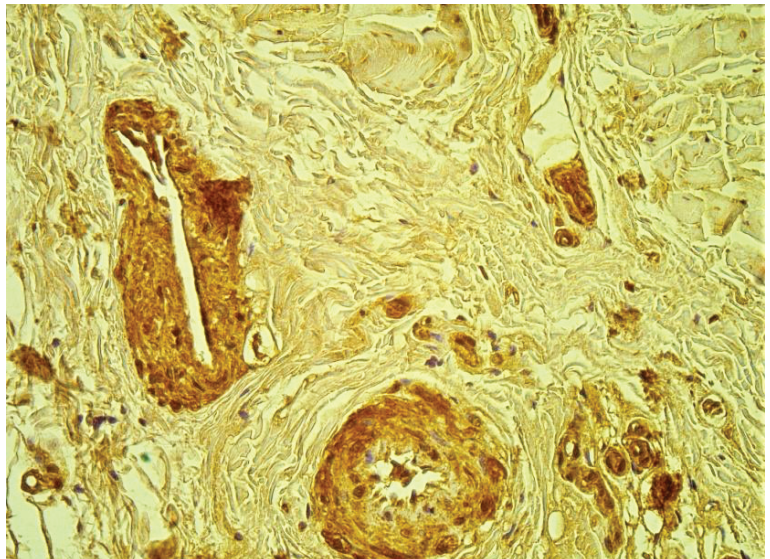

Fig. 7. Moderate number of VEGF positive endotheliocytes seen in musculus semitendinosus peritenon in 31 years old patient material. VEGF IMH, X250

Table 1. The relative number of VEGF, PGP9.5, collagen I and III positive structures observed in musculus semitendinosus tendon

\begin{tabular}{|c|c|c|c|c|c|c|}
\hline N. & Age & Gender & VEGF & PGP 9.5 & $\begin{array}{c}\text { Collagen } \\
\text { III }\end{array}$ & $\begin{array}{c}\text { Collagen } \\
\text { I }\end{array}$ \\
\hline 1 & 26 & $\mathrm{M}$ & $0 /+$ & + & + & ++ \\
\hline 2 & 31 & $\mathrm{M}$ & $++/+++$ & ++ & $++/+++$ & $0 /+$ \\
\hline 3 & 38 & $\mathrm{M}$ & $0 /+$ & 0 & $0 /+$ & ++ \\
\hline 4 & 55 & $\mathrm{M}$ & +++ & ++ & $++/+++$ & + \\
\hline
\end{tabular}

\section{REFERENCES}

1. Ackermann PW, Ahmed M, Kreicbergs A. Early nerve regeneration after achilles tendon rupturea prerequisite for healing? A study in the rat. // Journal of orthopaedic research, 2002; 20(4):849-856

2. Boesen AP, Dideriksen K, Couppé C et. al. Tendon and skeletal muscle matrix gene expression and functional responses to immobilisation and rehabilitation in young males: effect of growth hormone administration. // The Journal of physiology, 2013; 591(23):6039-6052

3. Buono AD, Oliva F, Longo UG et al. Metalloproteases and rotator cuff disease // Journal of Shoulder and Elbow Surgery, 2012; 21 (2): 200-208

4. Fabregat A, Jupe S, Matthews L et al. The Reactome Pathway Knowledgebase. // Nucleic acids research, 2018; 46(D1):D649-D655

5. Gobbi A, Francisco R. Hamstring Anterior Cruciate Ligament Reconstruction With a Quadrupled or Tripled Semitendinosus Tendon Graft // Prodromos CC. The Anterior Cruciate Ligament: Reconstruction and Basic Science, 2nd ed. Philadelphia: Elsevier; 2018; 103-106

6. Grewal N, Thornton GM, Behzad H et al. Accumulation of oxidized LDL in the tendon tissues of C57BL/6 or apolipoprotein E knock-out mice that consume a high fat diet: potential impact on tendon health. // PloS one, 2014; 9(12):el 14214

7. Hernandez P, Buller D, Mitchell T et al. Severe Burn-Induced Inflammation and Remodeling of Achilles Tendon in a Rat Model. // Shock (Augusta, Ga.), 2018; 50(3):346-350

8. Holladay C, Abbah SA, O'Dowd C et al. Preferential tendon stem cell response to growth factor supplementation. // Journal of tissue engineering and regenerative medicine, 2016; 10(9):783-798

9. Howell K, Chien C, Bell R et al. Novel Model of Tendon Regeneration Reveals Distinct Cell Mechanisms Underlying Regenerative and Fibrotic Tendon Healing // Scientific Reports, 2017; 7:45238

10. The female ACL: Why is it more prone to injury? // Journal of Orthopaedics, 2016; 13 (2):Al-A4

11. Leal MF, Caires Dos Santos L, Martins de Oliveira A et al. Epigenetic regulation of metalloproteinases and their inhibitors in rotator cuff tears // PloS one, 2017; 12(9):e0184141

12. Magnusson SP, Langberg $H$, Kjaer $M$. The pathogenesis of tendinopathy: balancing the response to loading // Nature Reviews Rheumatology, 2010; 6:262-268

13. Marqueti RC, Durigan JLQ, Oliveira AJS et al. Effects of aging and resistance training in rat tendon remodelling // FASEB journal, 2017; 32(1):353-368

14. Millar NL, Gilchrist DS, Akbar Metal. MicroRNA29a regulates IL-33-mediated tissue remodelling in tendon disease// Nature Communications, 2015; 6:6774

15. Miller RH, Azar FM. Knee Injuries // Azar FM, Beaty JH, Canale ST. Campbell's Operative Orthopaedics, 13th ed. Philadelphia: Elsevier; 2017; 2121-2297

16. Neeraj S. International Epidemiology of Anterior Cruciate Ligament Injuries // Orthopedic Research Online Journal, 2018; 1(5)

17. Osiecka-Iwan A, Moskalewski S, Kosowska A et al. Influence of cartilage interstitial fluid on gene expression in cruciate ligament fibroblasts // Experimental and therapeutic medicine, 2018; 15(1):387-392

18. Phillips BB, Mihalko MJ. Arthroscopy of the Lower Extremity // Campbell's Operative Orthopaedics, 13th ed. Philadelphia: Elsevier; 2017; 2486-2566

19. Pilmane M, Rumba I, Sundler F et al. Patterns of distribution and occurrence of neuroendocrine elements in lungs of humans with chronic lung diseases // Proc. of the Latvian Academy of Sciences, Section B, 1998, 52:144-152

20. Prodromos CC, Finkle SM, Han Y et al. Incidence of Anterior Cruciate Ligament Injury as a Function of Gender, Sport, and Injury-Reduction Programs // Prodromos CC. The Anterior Cruciate Ligament: Reconstruction and Basic Science, 2nd ed. Philadelphia: Elsevier; 2018; 29-40,

21. Sabatelli P, Sardone F, Traina F et al. TGF- $\beta 1$ differentially modulates the collagen VI $\alpha 5$ and 
a6 chains in human tendon cultures // Journal of biological regulators and homeostatic agents, 2016; 30(4 Suppl 1):107-113

22. Sahemey R, Malliaras $P$, Lewis JS et al. Tendinopathic supraspinatus tenocytes may have a neuroendocrine-like function, secreting CGRP, SP and VEGF: a pilot immunohistochemistry study // Journal of biological regulators and homeostatic agents, 2016; 30(4 Suppl 1):219-227

23. Snedeker JG, Foolen J. Tendon injury and repair A perspective on the basic mechanisms of tendon disease and future clinical therapy // Acta Biomaterialia, 2017; 63, 18-36

24. Tempfer H, Kaser-Eichberger A, Lehner C et al. Bevacizumab Improves Achilles Tendon Repair in a Rat Model // Cellular physiology and biochemistry, 2018; 46(3):1148-1158

25. Tohyama H, Yasuda K, Kitamura Y et al. The changes in mechanical properties of regenerated and residual tissues in the patellar tendon after removal of its central portion// Clinical Biomechanics, 2003; 18(8):765-772
26. Tosounidis T, Hadjileontis C, Georgiadis M et al. The tendon of the long head of the biceps in complex proximal humerus fractures: A histological perspective // Injury, 2010; 41(3): 273-278

27. Vavvas DG, Thanos A, Patel AV et al. Basic Mechanisms of Pathologic Retinal and Choroidal Angiogenesis // Schachat AP, Ryan's Retina, 6th ed. Philadelphia: Elsevier; 2018; 638-655

28. Wei X, Mao Z, Hou Y et al. Local administration of TGF $\beta$-1/VEGF165 gene-transduced bone mesenchymal stem cells for Achilles allograft replacement of the anterior cruciate ligament in rabbits // Biochemical and biophysical research communications, 2011; 406(2):204-210

29. Xu Y, Bonar F, Murrell GA. Neoinnervation in rotator cuff tendinopathy // Sports medicine and arthroscopy review, 2011 ; 9(4):354-359

30. Zhang C, Liu YJ. Biomechanic and histologic analysis of fibroblastic effects of tendon-to-bone healing by transforming growth factor $\beta 1$ (TGF- $\beta 1$ ) in rotator cuff tears // Acta cirurgica brasileira, 2017; 32(12):1045-1055

\section{Address:}

Janis Davis Osipovs

Institute of Anatomy and Anthropology

Kronvalda bulv. 9, Riga, Latvia, LV - 1010

janis.davis.osipovs@gmail.com 